blast niche? Finally, studies are needed to better characterize the biological effects of the osteoblast niche on the survival, quiescence, and sensitivity to chemotherapy of cancer cells. Ultimately, identification of the niche signals that regulate cancer cell phenotype may provide targeted strategies to render metastatic bone cancers more susceptible to chemotherapy.

Address correspondence to: Daniel C. Link, Division of Oncology, Department of Medicine, 660 S. Euclid Avenue, Campus Box 8007, St. Louis, Missouri 63110, USA. Phone: 314.362.8771; Fax: 314.362.9333; E-mail:dlink@dom.wustl.edu.

1. Roodman GD. Mechanisms of bone metastasis. NEngl J Med. 2004;350(16):1655-1664.

2. Shiozawa Y, et al. Human prostate cancer metastases target the hematopoietic stem cell niche to establish footholds in mouse bone marrow. J Clin Invest. 2011;121(4):1298-1312.

3. Kiel MJ, Yilmaz OH, Iwashita T, Terhorst C, Morrison SJ. SLAM family receptors distinguish hematopoietic stem and progenitor cells and reveal endothelial niches for stem cells. Cell. 2005;121(7):1109-1121.
4. Nilsson SK, Johnston HM, Coverdale JA. Spatial localization of transplanted hemopoietic stem cells: inferences for the localization of stem cell niches. Blood. 2001;97(8):2293-2299.

5. Mendez-Ferrer S, et al. Mesenchymal and haematopoietic stem cells form a unique bone marrow niche. Nature. 2010;466(7308):829-834.

6. Sugiyama T, Kohara H, Noda M, Nagasawa T. Maintenance of the hematopoietic stem cell pool by CXCL12-CXCR4 chemokine signaling in bone marrow stromal cell niches. Immunity. 2006; 25(6):977-988.

7. Omatsu Y, et al. The essential functions of adipoosteogenic progenitors as the hematopoietic stem and progenitor cell niche. Immunity. 2010;33(3):387-399.

8. Calvi LM, et al. Osteoblastic cells regulate the haematopoietic stem cell niche. Nature. 2003; 425(6960):841-846

9. Visnjic D, Kalajzic Z, Rowe DW, Katavic V, Lorenzo J, Aguila HL. Hematopoiesis is severely altered in mice with an induced osteoblast deficiency. Blood. 2004; 103(9):3258-3264.

10. Cashman J, Clark-Lewis I, Eaves A, Eaves C. Stromal-derived factor 1 inhibits the cycling of very primitive human hematopoietic cells in vitro and in NOD/SCID mice. Blood. 2002;99(3):792-799.

11. Peled A, et al. Dependence of human stem cell engraftment and repopulation of NOD/SCID mice on CXCR4. Science. 1999;283(5403):845-848.

12. Christopher MJ, Liu F, Hilton MJ, Long F, Link DC. Suppression of CXCL12 production by bone marrow osteoblasts is a common and critical pathway for cytokine-induced mobilization. Blood.
2009;114(7):1331-1339.

13. Liles WC, et al. Mobilization of hematopoietic progenitor cells in healthy volunteers by AMD3100, a CXCR4 antagonist. Blood. 2003; 102(8):2728-2730

14. Arai F, et al. Tie2/angiopoietin-1 signaling regulates hematopoietic stem cell quiescence in the bone marrow niche. Cell. 2004;118(2):149-161.

15. Yoshihara H, et al. Thrombopoietin/MPL signaling regulates hematopoietic stem cell quiescence and interaction with the osteoblastic niche. Cell Stem Cell. 2007;1(6):685-697.

16. Duncan AW, et al. Integration of Notch and Wnt signaling in hematopoietic stem cell maintenance. Nat Immunol. 2005;6(3):314-322.

17. Balkwill F. The significance of cancer cell expression of the chemokine receptor CXCR4. Semin Cancer Biol. 2004;14(3):171-179.

18. Liang $Z$, et al. Inhibition of breast cancer metastasis by selective synthetic polypeptide against CXCR4. Cancer Res. 2004;64(12):4302-4308.

19. Huang EH, et al. A CXCR4 antagonist CTCE-9908 inhibits primary tumor growth and metastasis of breast cancer. J Surg Res. 2009;155(2):231-236.

20. Morgan TM, et al. Disseminated tumor cells in prostate cancer patients after radical prostatectomy and without evidence of disease predicts biochemical recurrence. Clin Cancer Res. 2009;15(2):677-683.

21. Nervi $B$, et al. Chemosensitization of acute myeloid leukemia (AML) following mobilization by the CXCR4 antagonist AMD3100. Blood. 2009;113(24):6206-6214.

\title{
Finding a needle in a haystack: whole genome sequencing and mutation discovery in murine models
}

\author{
Raajit K. Rampal and Ross L. Levine \\ Human Oncology and Pathogenesis Program and Leukemia Service, Department of Medicine, \\ Memorial Sloan-Kettering Cancer Center, New York, New York, USA.
}

\begin{abstract}
Acute promyelocytic leukemia (APL) is a malignancy of the bone marrow, in which there is a deficiency of myeloid cells and an excess of immature cells called promyelocytes. APL is most commonly caused by a translocation (15:17) and expression of the promyelocytic leukemia and the retinoic receptor $\alpha$ (PML-RARA) fusion product; however, the events that cooperate with PML-RARA in APL pathogenesis are not well understood. In this issue of the JCI, Wartman and colleagues use an innovative approach to find other relevant mutations in APL. They performed whole genome sequencing and copy number analysis of a well-characterized APL mouse model to uncover somatic mutations in Jak1 and lysine (K)-specific demethylase 6A (Kdm6a, also known as $U t x)$ in mice with APL and validated the ability of Jak1 mutations to cooperate with PML-RARA in APL. The findings implicate the JAK/STAT pathway in the pathogenesis of APL and illustrate the power of whole genome sequencing to identify novel disease alleles in murine models of disease.
\end{abstract}

Conflict of interest: The authors have declared that no conflict of interest exists.

Citation for this article: J Clin Invest. 2011; 121(4):1255-1258. doi:10.1172/JCI57200.
Acute promyelocytic leukemia (APL) is a clinically and molecularly distinct subtype of acute myeloid leukemia that is distinguished by a recurrent chromosom- al translocation fusing chromosomes 15 and 17 . The $t(15: 17)$ translocation results in the fusion of the promyelocytic leukemia $(P M L)$ gene and the retinoic receptor $\alpha(R A R A)$ gene (PML-RARA). The PMLRARA fusion protein is thought to contribute to APL pathogenesis by dimerizing and binding DNA and repressing the transcription of RARA target genes through recruitment of corepressors. More recent work indicates that PMLRARA also is able to bind to alternate DNA sites and to interact with chromatin remodeling complexes involved in stem cell maintenance and that the PMLRARA protein undergoes posttranslational modifications (sumoylation and phosphorylation) that are required for APL initiation $(1,2)$. A detailed understanding of the role of the PML-RARA 
Table 1

JAK mutations in human malignancies

\begin{tabular}{|c|c|c|c|}
\hline JAK & Mutation & Human disease & Ref. \\
\hline \multirow[t]{19}{*}{ JAK1 } & T478S & AML & 15 \\
\hline & V623A & AML & 15 \\
\hline & G871E & Uterine leiomyosarcoma & 18 \\
\hline & V658F & T-ALL, APL, pediatric ALL & $14,16,10$ \\
\hline & L783F & T-ALL & 16 \\
\hline & T782M & NSCLC & 16 \\
\hline & $\mathrm{H} 647 \mathrm{Y}$ & Breast cancer & 16 \\
\hline & $162 \mathrm{~V}$ & B-ALL, T-ALL & 17 \\
\hline & K204M & B-ALL & 17 \\
\hline & A634D & B-ALL, T-ALL & 17 \\
\hline & $\mathrm{R} 724 \mathrm{H}$ & B-ALL, T-ALL & 17 \\
\hline & R360W & T-ALL & 17 \\
\hline & S512L & T-ALL & 17 \\
\hline & R879S & T-ALL & 17 \\
\hline & R879C & T-ALL & 17 \\
\hline & $\mathrm{R} 879 \mathrm{H}$ & T-ALL & 17 \\
\hline & L653F & Pediatric ALL & 17 \\
\hline & L624_R629>W & Pediatric ALL & 14 \\
\hline & S646F & Pediatric ALL & 14 \\
\hline \multirow[t]{11}{*}{ JAK2 } & V617F & MPN & 18 \\
\hline & K539L & PV & 18 \\
\hline & T875N & AML & 18 \\
\hline & del682-686 & ALL & 18 \\
\hline & R683G & Pediatric ALL/Down syndrome ALL & 14 \\
\hline & R683S & Pediatric ALL & 14 \\
\hline & $1682 \mathrm{~F}$ & Pediatric ALL & 14 \\
\hline & QGinsR683 & Pediatric ALL/Down syndrome ALL & 14 \\
\hline & R867Q & Pediatric ALL & 14 \\
\hline & D873N & Pediatric ALL & 14 \\
\hline & P933R & Pediatric ALL & 14 \\
\hline \multirow[t]{5}{*}{ JAK3 } & A572V & AML & 18 \\
\hline & V7221 & AML & 18 \\
\hline & P132T & AML & 18 \\
\hline & V715I & Breast cancer & 16 \\
\hline & S789P & Pediatric ALL & 14 \\
\hline
\end{tabular}

B-ALL, B cell ALL; NSCLC, non-small cell lung cancer; PV, polycythemia vera; T-ALL, T cell ALL.

oncogenic fusion in APL pathogenesis has allowed investigators to elucidate the molecular basis by which retinoic acid and arsenic trioxide offer dramatic efficacy in human patients with APL. Importantly, current therapeutic approaches combining retinoic acid/arsenic with anthracyclines allow as many of $90 \%$ of patients with APL to be cured (1).

The incidence of APL is reported to be constant over a human life span, which has led to models suggesting there is a single genetic rate-limiting step involved in the development of APL (3). However, mouse models of APL suggest that PMLRARA expression in vivo leads to APL with long latency and incomplete penetrance. This suggests that the acquisition of other genetic alterations in addition to PML-RARA contributes to the develop- ment of APL (4). Indeed, data from transgenic mouse models expressing PMLRARA indicate that mice that progress to APL acquire additional genetic alterations (4-6). Studies in patients with APL have also demonstrated the occurrence of activating FLT3 mutations and NRAS, KRAS, and MYC mutations (2). Collectively, these studies indicate that other genetic alterations may contribute to APL pathogenesis, even if PML-RARA is the diseaseinitiating event.

\section{The utility of mouse models of APL}

Although there exist key genetic and biologic differences in the development of malignancies between mice and humans, mouse models of APL have proven to be very useful in both modeling APL pathogenesis and in investigating specific therapies. Such models have been used to elucidate the effects and mechanisms of retinoic acid and arsenic trioxide therapy, with mouse APL cells demonstrating similar in vivo responses to both compounds when compared to human APL cells (7).

Several approaches have been used to generate mouse APL models, including transgenic PML-RARA expression, xenograft models, and adoptive transfer strategies. Transgenic models placing $P M L-$ RARA under the control of cathepsin G or migration inhibitory factor-related protein 8 (MRP8) promoters resulted in APL; however, in each case, APL developed with relatively long latency (6 months or longer) with incomplete penetrance (8). These data suggest additional mutations are required for the development of APL. The role of cooperating disease alleles in APL pathogenesis is underscored by mutational studies of primary human APL samples. Candidate gene studies have shown that mutations in NRAS, KRAS, FLT3-internal tandem duplication (FLT3-ITD), and FLT3-tyrosine kinase domain (FLT3-TKD) as well as trisomy 8 are observed in a subset of patients who present with APL (2). The functional significance of the Kras mutation in particular was elucidated by Chan et al., who found that coexpressing oncogenic Kras from the endogenous Ras locus with PML-RARA resulted in APL with shorter latency and near complete penetrance (9). Taken together, the human and murine genetic data suggest that additional disease alleles cooperate with PML-RARA in APL pathogenesis.

To elucidate potential cooperating events in murine APL models, previous studies performed karyotypic analysis. Zimonjic et al. used spectral karyotype analysis to identify recurrent abnormalities in murine APL cells, including interstitial or terminal deletion of one copy of chromosome 2, gains of chromosome 15 , and loss of chromosome 11, X, and $\mathrm{Y}(5)$. Le Beau et al. performed spectral karyotyping analysis in hMRP8-PML-RARA mice and identified trisomies 8, 15, and 16 and monosomies $\mathrm{X}$ or $\mathrm{Y}$ as recurrent somatic alterations in murine APL cells (4). These results collectively indicate that PML-RARA fusion is necessary but not sufficient to produce APL in murine transgenic models. However, in the majority of humans with APL and in most murine APL models, the identity of cooperating disease alleles has not been revealed. 


\section{Whole genomic sequencing}

In this issue of the JCI, Wartman and colleagues used massive parallel DNA sequencing in an effort to perform systematic mutational analysis of the murine APL genome (10). Until recently, whole genome sequencing of primary murine and human tumors was not feasible due to cost and the requirement for large amounts of tumor material. However, these limitations have largely been overcome due to improved sequencing technology and analytic tools (11). Indeed, previous efforts by the current investigators using whole genome sequencing in human patients with acute myelogenous leukemia (AML) has allowed them to identify novel clinically and biologically relevant AML mutations, demonstrating the power of massive parallel sequencing $(11,12)$.

Wartman et al. used an innovative strategy to find additional mutations in this APL model (10). An inbred mouse strain was used in an attempt to reduce the number of variants, as many of the variants found in sequencing a murine genome may not be relevant to disease pathogenesis. Mice expressing the PML-RARA transgene under the control of the murine cathepsin $\mathrm{G}$ promoter were backcrossed to the Black 6/Taconic background for 10 generations. These mice developed an APL-like disease with a relatively long latency (9-12 months), suggesting the acquisition of additional genetic events is required for APL development in this model. In previous human studies, tumor whole genome sequencing data were compared with sequencing data from matched germline DNA to assess whether candidate mutations were present in the germline or were bona fide somatic mutations acquired during tumorigenesis. In contrast, here the authors compared the spectrum of single nucleotide variants present in murine APL cells with a sequenced genome of the initial mouse strain. An alternative, and perhaps more discriminating, strategy might have compared the APL mutational data with DNA from littermate controls or compared the murine APL genome with hematopoietic DNA from the same mouse from an earlier time point, before APL development.

Six nonsynonymous mutations were identified and validated as being present in the APL genome; the authors then performed secondary mutational analysis of 89 additional mouse APL samples for these 6 mutations. Importantly, this approach allowed them to identify that one mutation, Jak1 V658F, was present as a recurrent alteration in murine APL. Of note, the Jak1 V658F mutation occurs at the homologous position to JAK2 V617, which is commonly mutated in patients with myeloproliferative neoplasms (MPNs) (13), and has been observed previously in patients with high-risk acute lymphoblastic leukemia (ALL) (14). They then ectopically expressed Jak 1 V658F in mCG-PML-RARA bone marrow, followed by transplantation into lethally irradiated recipients, which resulted in a short latency, completely penetrant APL phenotype. In addition, they also performed highresolution copy number analysis of the murine tumors and identified a somatic deletion of a histone demethylase, lysine (K)-specific demethylase 6A (Kdm6a, also known as $U t x)$, in the same murine APL genome, a deletion also observed in human APL. However, its functional contribution to APL pathogenesis and to APL development in this mouse model has not been elucidated.

\section{Mutations in the JAK/STAT pathway and targeted therapies}

The JAKs are involved in the transduction of cytokine receptor signaling. These kinases (JAK1, JAK2, JAK3, and Tyk2) bind to the cytosolic domains of cytokine receptors. Conformational changes occur in the cytokine receptor as a result of binding to cytokines, allowing them to recruit JAKs. Somatic mutations in JAKs have been described in human malignancy (Table 1). Most notable has been the elucidation of the role of JAK2 V617F mutation in MPN pathogenesis. Somatic JAK1 mutations have been recently described in AML $(15,16)$ and in ALL (17). These genetic observations have led to the development of JAK inhibitors, including inhibitors with JAK1 inhibitory activity; these agents have now entered late-stage clinical trials in patients with MPN. Here, the investigators used a pan-JAK inhibitor in the context of a methylcellulose assay to show that JAK1 inhibition exhibited similar efficacy to all-trans retinoic acid (a standard treatment for APL) in reducing APL colony formation. Furthermore, the use of the pan-JAK inhibitor decreased STAT5 phosphorylation, indicating on-target effects with regards to the JAK/STAT pathway. Collectively, these data indicate a role for the JAK/STAT pathway in the pathogenesis of APL in this model that extends beyond the presence of a JAK mutation. The role of the JAK/STAT pathway in APL and in other AML subtypes thus warrants further investigation. In addition, whether JAK1-mutant APL constitutes a specific clinicopathologic subtype of APL with prognostic or therapeutic relevance remains to be delineated.

\section{Conclusions}

Wartman and colleagues successfully employed an elegant use of whole genome sequencing as a dragnet to ensure broad coverage of the genome. It is possible that this strategy may not identify all relevant disease alleles, due to the sequencing approach, the analytic platform, or the presence of large mutations that may be missed by short-read parallel sequencing. However, the strength of this approach is demonstrated in this report, as the authors show how whole genome sequencing can be used to provide pathogenetic insight in murine cancer models. We predict that subsequent utilization of this approach will allow investigators to add further to the list of recurrent driver mutations that contribute to malignant transformation.

\section{Acknowledgments}

R.K. Rampal is supported by the Memorial Sloan-Kettering Cancer Center Clinical Scholars Program. R.L. Levine is a Geoffrey Beene Junior Faculty Chair at the Memorial Sloan-Kettering Cancer Center.

Address correspondence to: Ross L. Levine, Memorial Sloan-Kettering Cancer Center, 1275 York Avenue, Box 20, New York, New York 10065, USA. Phone: 646.888.2767; Fax: 646.422.0856; E-mail: leviner@mskcc.org.

1. de The H, Chen Z. Acute promyelocytic leukaemia: novel insights into the mechanisms of cure. Nat Rev Cancer. 2010;10(11):775-783.

2. Akagi $\mathrm{T}$, et al. Hidden abnormalities and novel classification of $\mathrm{t}(15 ; 17)$ acute promyelocytic leukemia (APL) based on genomic alterations. Blood. 2009;113(8):1741-1748

3. Vickers M, Jackson G, Taylor P. The incidence of acute promyelocytic leukemia appears constant over most of a human lifespan, implying only one rate limiting mutation. Leukemia. 2000;14(4):722-726.

4. Le Beau MM, Bitts S, Davis EM, Kogan SC. Recurring chromosomal abnormalities in leukemia in PMLRARA transgenic mice parallel human acute promyelocytic leukemia. Blood. 2002;99(8):2985-2991.

5. Zimonjic DB, Pollock JL, Westervelt P, Popescu NC, Ley TJ. Acquired, nonrandom chromosomal abnormalities associated with the development of acute promyelocytic leukemia in transgenic mice. Proc Natl Acad Sci U S A. 2000;97(24):13306-13311. 6. Le Beau MM, Davis EM, Patel B, Phan VT, Sohal J, 
Kogan SC. Recurring chromosomal abnormalities in leukemia in PML-RARA transgenic mice identify cooperating events and genetic pathways to acute promyelocytic leukemia. Blood. 2003;102(3):1072-1074.

7. Lallemand-Breitenbach V, Zhu J, Kogan S, Chen Z, de The H. Opinion: how patients have benefited from mouse models of acute promyelocytic leukaemia. Nat Rev Cancer. 2005;5(10):821-827.

8 . Kogan SC. Mouse models of acute promyelocytic leukemia. Curr Top Microbiol Immunol. 2007;313:3-29.

9. Chan IT, et al. Oncogenic K-ras cooperates with PMLRAR alpha to induce an acute promyelocytic leukemia-like disease. Blood. 2006;108(5):1708-1715.
10. Wartman LD, et al. Sequencing a mouse acute promyelocytic leukemia genome reveals genetic events relevant for disease progression. J Clin Invest. 2011; 121(4):1445-1455

11. Ley TJ, et al. DNMT3A mutations in acute myeloid leukemia. N Engl J Med. 2010;363(25):2424-2433.

12. Mardis ER, et al. Recurring mutations found by sequencing an acute myeloid leukemia genome. N Engl J Med. 2009;361(11):1058-1066.

13. Campbell PJ, Green AR. The myeloproliferative disorders. N Engl J Med. 2006;355(23):2452-2466.

14. Mullighan CG, et al. JAK mutations in high-risk childhood acute lymphoblastic leukemia. Proc Natl
Acad Sci U S A. 2009;106(23):9414-9418.

15. Xiang $Z$, et al. Identification of somatic JAK1 mutations in patients with acute myeloid leukemia. Blood. 2008;111(9):4809-4812.

16. Jeong EG, et al. Somatic mutations of JAK1 and JAK3 in acute leukemias and solid cancers. Clin Cancer Res. 2008;14(12):3716-3721.

17. Flex E, et al. Somatically acquired JAK1 mutations in adult acute lymphoblastic leukemia. J Exp Med. 2008;205(4):751-758

18. Constantinescu SN, Girardot M, Pecquet C. Mining for JAK-STAT mutations in cancer. Trends Biochem Sci. 2008;33(3):122-131.

\title{
Dietary nitrate, nitric oxide, and restenosis
}

\author{
John P. Cooke and Yohannes T. Ghebremariam
}

Stanford Cardiovascular Institute, Stanford, California, USA.

\begin{abstract}
Endothelium-derived NO controls the contractility and growth state of the underlying vascular smooth muscle cells and regulates the interaction of the vessel wall with circulating blood elements. Acute injury of the vessel wall denudes the endothelial lining, removing homeostatic regulation and precipitating a wave of events leading to myointimal hyperplasia. In this issue of the JCI, Alef and colleagues provide evidence that in the injured vessel wall, the disruption of the NOS pathway is countered by induction of xanthine oxidoreductase, an enzyme capable of producing NO from nitrite. In addition, they link low dietary nitrite levels to increased severity of myointimal hyperplasia following vessel injury in mice.
\end{abstract}

\section{The role of endothelium-derived NO in vascular homeostasis}

The endothelium is a diaphanous film of tissue comprising a single-cell monolayer that lines the luminal surface of all blood vessels. Despite its fragility, this delicate membrane exerts dramatic control over vascular homeostasis. Endotheliumderived factors control the contractility and growth state of the underlying VSMCs, and regulate the interaction of the vessel wall with circulating blood elements. NO is arguably the most influential of these paracrine substances.

$\mathrm{NO}$ is derived from the metabolism of L-arginine by NOS. NO activates soluble guanylate cyclase to produce cGMP, which participates in signaling pathways involving phosphorylation (1). Furthermore, NO regulates the activities of enzymes and signaling proteins by S-nitrosylation of thiol moieties (2). The endothelial synthesis of this potent molecule is highly regulated, in response to hemodynamic (e.g.,

Conflict of interest: John P. Cooke is an inventor on Stanford University patents related to therapeutic modulation of the NOS pathway.

Citation for this article: J Clin Invest. 2011; 121(4):1258-1260. doi:10.1172/JCI57193. shear stress) as well as humoral (e.g., serotonin) factors (3). Endothelium-derived NO suppresses platelet adhesion, leukocyte infiltration, and VSMC proliferation and migration (3). Finally, endotheliumderived NO suppresses the endothelial expression of adhesion molecules and chemokines that would otherwise initiate vascular inflammation (3). Cardiovascular risk factors (e.g., hypercholesterolemia, diabetes mellitus) impair the synthesis and bioactivity of endothelium-derived NO $(4,5)$. Their adverse effect on the NOS pathway is mediated by asymmetric dimethylarginine (ADMA; the endogenous antagonist of NO synthesis) and by the vascular generation of superoxide anion $\left(\mathrm{O}_{2}{ }^{-}\right)$, which degrades $\mathrm{NO}$ to peroxynitrite anion $\left(\mathrm{ONOO}^{-}\right)(4,5)$.

\section{Myointimal hyperplasia: response to vascular injury and loss of endothelial homeostasis}

Acute injury of the vessel wall (as occurs with balloon angioplasty) denudes the endothelium, fractures the internal elastic lamina, and damages the underlying VSMCs, removing homeostatic regulation and precipitating a wave of events that can lead to myointimal hyperplasia. Plate- lets adhere to collagen in the damaged vessel wall, and secrete PDGF and other proliferative factors. Infiltrating immune cells secrete inflammatory cytokines that contribute to cellular proliferation, and injured VSMCs release FGF. Vascular progenitor cells circulating in the blood may also contribute to the injury response (6). In the absence of the moderating influence of the endothelium, VSMCs from the media proliferate and migrate into the intimal space, and secrete extracellular matrix. This phenotypic modulation is transcriptionally regulated by CArG box DNA sequences within promoter chromatin of VSMC genes (7).

At the site of vascular injury, arginase is induced, converting arginine to urea and ornithine (8). Ornithine serves as a precursor for polyamines that are known to stimulate VSMC proliferation. Although the endothelial source of $\mathrm{NO}$ generation is lost, the activated VSMCs and the infiltrating immune cells express the inducible form of NOS (iNOS). Unfortunately, this enzyme is an imperfect replacement for endothelial NO synthase (eNOS). Unlike eNOS, which produces small amounts of $\mathrm{NO}$ in a compartmentalized and regulated fashion, iNOS is constitutively active, with a $V_{\max }$ that is a thousand-fold that of eNOS. As a result, iNOS quickly outstrips the reduced supply of arginine and donates electrons to oxygen, generating $\mathrm{O}_{2}^{-}$(9). Superoxide anion combines with $\mathrm{NO}$ to form the cytotoxic radical $\mathrm{ONOO}^{-}$. Subsequent activation of oxidant-sensitive transcriptional pathways increases VSMC proliferation and migration (10), contributing to the myointimal lesion. 